\title{
Evaluation of further reduced resolution depth coding for stereoscopic 3D video
}

\begin{abstract}
This paper presents the results and analysis of the objective and subjective quality evaluations of Further Reduced Resolution Depth Coding (FRRDC) method for stereoscopic 3D video. FRRDC is developed based on the Scalable Video Coding (SVC) reference software and the result are objectively evaluated using rate distortion curve and subjectively evaluated using LCD and auto-stereoscopic video displays. FRRDC uses the Down-Sampling and UpSampling (DSUS) method of the depth data of the stereoscopic 3D video. The emergence of numerous auto-stereoscopic displays in the market confirms the growth of 3DTV services. It is essential that the coding method of stereoscopic 3D videos produces high quality 3D videos on both stereoscopic displays and emerging auto-stereoscopic 3D video displays to ensure the interoperability and compatibility among all the different display devices. In this paper, the stereoscopic 3D videos are compressed using the H.264/SVC codec with Reduced Resolution Depth Coding (RRDC) and compared with H.264/SVC-FRRDC. The experimental results indicate good 3D depth perception of FRRDC on both stereoscopic and auto-stereoscopic display devices with lesser bit rates compared to H.264/SVC-RRDC.
\end{abstract}

Keyword: Reduced resolution depth coding; Stereoscopic 3D video 